First publ. in: Zeitschrift für Physik B - Condensed Matter 87 (1992), pp. $43-49$

\title{
$\beta$ relaxation theory for simple systems: some addenda
}

\author{
M. Fuchs, W. Götze ${ }^{\star}$, S. Hildebrand, and A. Latz \\ Physik-Department, Technische Universität München, W-8046 Garching, Federal Republic of Germany
}

Received October 21, 1991

The evolution of the tagged particle probability density for a hard sphere system is evaluated within the $\beta$-relaxation window. Relaxation curves obtained by molecular dynamics studies by Barrat, Hansen and Roux for a binary mixture are analyzed quantitatively with $\beta$-relaxation scaling formulae. The dynamical light scattering data obtained by Pusey and van Megen for colloidal suspensions are described by the combined $\alpha$-and $\beta$-relaxation scaling results. The range of validity of asymptotic expressions near a glass transition singularity is discussed for the Debye-Waller factor as a function of packing fraction. The applied theoretical formulae are those of the mode coupling theory for the liquid to glass transition.

\section{Introduction}

Supercooled glass forming liquids exhibit $\beta$-relaxation in the mesoscopic dynamical window. It describes slow relaxation for times $t$ exceeding the scale $t_{0}$ for microscopic transient motion and preceding the scale $\tau$ for $\alpha$-relaxation processes: $t_{0} \ll t \ll \tau[1]$. The mode coupling theory for liquid dynamics, to be abbreviated as MCT, provides a rather detailed description of those $\beta$-processes; for a review compare [2]. In this article some further MCT results for simple systems shall be reported together with comparisons of theoretical findings with some data.

The basic result of the MCT for the $\beta$-dynamics is the factorization theorem expressing the correlation function $\Phi_{X Y}(t)$ of a pair of variables $X, Y$ of even time inversion symmetry in terms of the $\beta$-correlator $G(t)$ [3]:

$\Phi_{X Y}(t)=f_{X Y}^{c}+h_{X Y} G(t)$.

Here $f_{X Y}^{c}$ and $h_{X Y}$ are equilibrium quantities, the nonergodicity parameter at the bifurcation singularity and

^ Also at: Max-Planck-Institut für Physik und Astrophysik, W-8000 München, FRG the critical amplitude respectively. The time dependence of all correlators is given by the same function $G$, which obeys the scaling law

$G(t)=c_{\sigma} g_{ \pm}\left(\frac{t}{t_{\sigma}}\right), \quad \sigma \gtrless 0$

The separation parameter $\sigma$ is a smpoth function of the control parameters specifying the equilibrium structure. $\sigma \gtrless 0$ characterizes the glass and liquid side, respectively, of the transition hypersurface. The latter is described by the implicit equation $\sigma=0$ [4]. If the system is driven by some coupling constant $\Gamma$, like packing fraction $\varphi$ or inverse temperature $1 / T$, one can write

$\sigma=C \frac{\Gamma-\Gamma_{c}}{\Gamma_{c}}$

where the smooth function $C(\Gamma)$ can be replaced in leading order by its value at the transition for $\sigma \rightarrow 0: C\left(\Gamma_{c}\right)>0$. The sensitive dependence of $\Phi$ on $\Gamma$ is described by the correlation scale $c_{\sigma}$ and the $\beta$-time scale $t_{\sigma}$, which follow power laws:

$c_{\sigma}=|\sigma|^{\frac{1}{2}}, \quad t_{\sigma}=t_{0}|\sigma|^{-\frac{1}{2 a}}$.

Here $t_{0}$ is a smooth function of $\Gamma$, which for $\sigma \rightarrow 0$ can be replaced by its value at the transition. The scale $t_{0}$ connects the mathematical time scale with the physical one. From mode coupling functionals one can calculate a number $\lambda$, the exponent parameter obeying $1 / 2 \leqq \lambda<1$. This number fixes the critical exponent $a$, obeying $0<a<0.4$, and the whole master function $g_{+}$. The latter can be evaluated easily [5]. The master functions describe the cross over from critical decay

$g_{ \pm}(\hat{t} \ll 1)=\frac{1}{\hat{t}^{a}}$ 
to a constant or to the von Schweidler decay respectively:

$g_{+}(\hat{t} \gg 1)=\frac{1}{\sqrt{1-\lambda}} ; \quad g_{-}(\hat{t} \gg 1)=-B \hat{t}^{b}$.

The von Schweidler exponent $b$ obeys $0<b \leqq 1$. The von Schweidler law is also a part of the $\alpha$-relaxation. This process obeys a scaling law with a different time scale $t_{\sigma}^{\prime}:$

$\Phi_{X Y}(t)=F_{X Y}\left(\frac{t}{t_{\sigma}^{\prime}}\right)$ for $\sigma \rightarrow-0, t_{\sigma}^{\prime} \rightarrow \infty$.

The master function $F_{X Y}$ is independent of $\sigma$ in lowest order. The $\alpha$-relaxation scale $t_{\sigma}^{\prime}$, which is equivalent to $\tau$ up to a factor $\tilde{B}$ of the order unity $t_{\sigma}^{\prime}=\widetilde{B}^{1 / b} \tau$, is connected to the $\beta$-scaling time by

$t_{\sigma}^{\prime}=|\sigma|^{-\frac{1}{2 b}} t_{\sigma}=|\sigma|^{-\gamma} t_{0} \quad$ with $\quad \gamma=\frac{1}{2 a}+\frac{1}{2 b}$.

The formulae (2-6) imply a series of predictions, which can be tested against experiments even if one has to use $\lambda$ as a fit parameter, as was shown first for some dielectric loss spectra of polymers [6].

The quoted results refer to the ideal transition as it results within the simplest version of the MCT. The extended theory incorporates thermal activated processes, which restore ergodic motion for all parameters. Formulae $(1,5)$ remain valied for $\sigma \approx 0$, however the correlator $G$ has to be generalized to a two parameter scaling law. As a result the cross over phenomena from the critical decay to the long time behaviour are more complicated [7]. These complications shall not be considered in the present paper.

\section{Tagged particle motion for the hard sphere system}

The hard sphere system, to be abbreviated as HSS, is the simplest model for condensed matter. It can be studied experimentally as colloidal suspension; it exhibits a liquid to glass transition in the supercompressed state $[8,9]$. The MCT predicts the known $\alpha$ - and $\beta$-relaxation patterns for the correlation of density fluctuations $\Phi_{q}(t)$ and tagged particle correlators $\Phi_{q}^{s}(t)$. They are defined as auto correlations of the density fluctuations $\rho_{q}(t)$ or tagged particle density fluctuations $\rho_{q}^{s}(t)=\exp \left(i \mathbf{q} \mathbf{r}_{s}(t)\right)$ respectively. Both functions can be measured by coherent or incoherent dynamical light scattering $[8,9]$. There is the perspective that the mentioned system provides a model for studies of the glass transition without complications due to chemical subtleties; it also provides a means of testing the MCT in all details. The so far achieved results are suggesting. The experimental value for the critical packing fraction $\varphi^{c}=0.560 \pm 0.005$ and the Debye-Waller factor at the transition $\bar{f}_{q}^{c}$ [9] do not deviate from the predictions [10] by more than $10 \%$. The measurements of $\Phi_{q}(t)$ for $q=q_{0}$ chosen at the peak of the structure factor could be interpreted reasonably well with the $\beta$-relaxation theory [11].
The $\beta$-dynamics for $\Phi_{q}(t)$ was discussed previously [12]. The relevant functions $f_{q}^{s c}$ and $h_{q}^{s}$ entering (1) for $\Phi_{q}^{s}(t)=f_{q}^{s c}+h_{q}^{s} G(t)$ have been evaluated recently [13]. Calculating a Fourier back transform one gets the tagged particle correlation function in ordinary space $\Phi_{r}^{s}(t)=\left\langle\rho^{s}(\mathbf{r}, t) \rho^{s}(\mathbf{r}=\mathbf{0}, t=0)\right\rangle$, which reads in the $\beta$-region

$\Phi_{r}^{s}(t)=F^{s c}(r)+H^{s}(r) G(t)$.

Function $P(r, t)=4 \pi r^{2} \Phi_{r}^{s}(t)$ has the following significance: It is the probability density to find a marked particle at time $t$ at the distance $r$, if it was located for $t=0$ at the origin $\mathbf{r}=\mathbf{0}$. In a normal liquid state one gets for $t \gg t_{0}$ hydrodynamic motion. Thus $P(r, t)$ can be obtained from the solution of the diffusion equation: $P^{H}(r, t)=\frac{4 \pi r^{2}}{(4 \pi D t)^{3 / 2}} \exp -\left(r^{2} / 4 D t\right)$ where $D$ denotes the diffusion constant. This function exhibits one maximum at position $r_{\max }$, which moves to larger values with increasing time: $r_{\max }=\sqrt{4 D t}$. Quite opposed to that, there are no correlations between temporal and spatial variations in the $\beta$-region. The motion is localized there; from (9) one derives

$$
P^{\beta}(r, t)=P^{c}(r)+k(r) G(t) .
$$

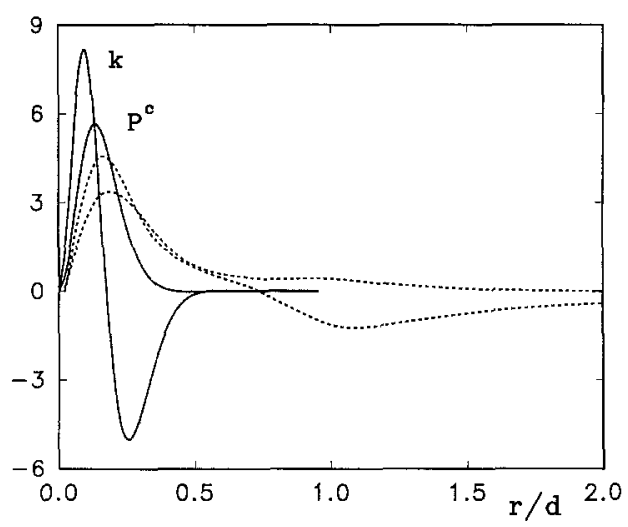

Fig. 1. $P^{c}(r)$ and $k(r)$ of the hard sphere system (solid lines) and qualitatively the experimental $P^{c}(r)$ and $k(r)$, which follow from MD simulations [14] (dotted lines). $d=\sqrt[3]{(6 / \pi n) \varphi}$ is the diameter of the hard spheres

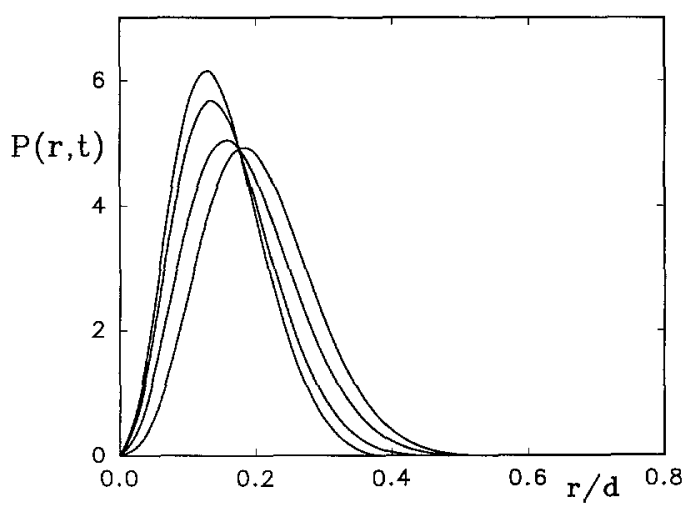

Fig. 2. $P\left(r, t_{i}\right)=P^{c}(r)+c_{\sigma} g\left(\hat{t}_{i}\right) k(r)$ for four representative values of $\hat{t}_{i}=t_{i} / t_{\sigma} i=1, \ldots, 4$. The curves with decreasing peak height from left to right correspond to $c_{\sigma}\left(\hat{t}_{i}\right)=0.08,0,-0.15,-0.3$ 
The calculated functions $P^{c}(r)$ and $k(r)$ are shown in Fig. 1. Notice that particle conservation implies the integral over $P(r, t)$ and $P^{c}(r)$ to be normalized to unity, so that $\int k(r) \mathrm{dr}=0$.

Figure 2 shows the evolution of the probability $P^{\beta}(r, t)$ for four values of the correlator $G_{i}, i=1 \ldots, 4$. For given separation parameter $\sigma$, these values can be connected with rescaled times $\hat{t}_{i}=t_{i} / t_{\sigma}$, using the known master functions $g_{ \pm}$of the HSS [11]. Notice that $\hat{t}_{2}=\hat{t}^{*}=0.608$ is the value, where $G_{-}$passes zero, so that $P^{\beta}\left(r, t_{2}\right)=P^{c}(r)$.

From Fig. 2 a physical picture of the $\beta$-relaxation within the MCT can be extracted. In dense systems the particles rattle in cages formed by the neighbouring particles. For $t_{0} \ll t \ll t *$ the mean square displacement is given by values close to the Lindemann ratio $r / d \approx 0.1$. Therefore the probability density $P(r, t)$ has its maximum at this distance. On cannot distinguish between glass and liquid state. With increasing time the particles explore their effective cages leading to the slight decrease of $P^{\beta}(r, \mathrm{t})$ for small $r$ as shown for $\hat{t}_{1}, \hat{t}_{2}$ in Fig. 2. For $\sigma \geqq 0$, i.e. in the glass, the probability arrests at the value $P^{c}(r)+\sqrt{\sigma /(1-\lambda)} h(r)$. For $\sigma=0$ this is shown by the curve $\hat{t}_{2}$.

In the liquid state the cages are becoming instable and the particles can leave them. In the von Schweidler regime the correlator $G(t)$ becomes negative. This leads to an increase of the probability for $r / d>0.17$ and to a decrease for $r / d<0.17$. This is shown in Fig. 2 for $\hat{t}_{3}, \hat{t}_{4}$. For $r / d=0.17$ the probability density remains constant, because $h(r)=0$. As a result the peak position moves out to higher values of $r$ and the height decreases. It is the $\alpha$-relaxation not described by (1) which ensures $P(r, t / \tau \rightarrow \infty) \rightarrow 0$ for $\sigma<0$. The curves in Fig. 2 have similarity to a diffusion process but due to the factorization property their nature is completely different. There is no coupling between space and time events. Detecting this factorization proves the experimental time window to be in the $\beta$-relaxation regime. Only a detailed analysis of the time dependence of $G(t)$ can teach whether the idealized MCT can be applied, especially whether $G(t)<0$ is due to the above mentioned von Schweidler law or due to similar decay laws [7] as caused by ergodicity restoring processes.

Using molecular dynamics simulations it has been possible to study $P(r, t)$ in a binary mixture of soft spheres [14]. For sphere diameters differing by a factor of 1.2 Roux et al. [14] observed two qualitatively different scenaria upon increasing the coupling constant $\Gamma$. Within the liquid regime where an $\alpha$-scaling law holds $P(r, t)$ exhibits one peak, which broadens and moves to larger $r$ with increasing time $t$. For times $t / \tau \gg 1$ long compared to the $\alpha$-relaxation time the diffusion law is found to apply to $P(r, t)$. The analysis of the $\alpha$-relaxation of a HSS in [13] shows that this is the qualitative picture expected in the $\alpha$-regime. For larger coupling constants $\Gamma \geqq 1.45, P(r, t)$ exhibits a two peak structure. One peak for $r / d \approx 0.19$ decreases with increasing time, while a second one appears at $r / d \approx 1$. In between these peaks all curves intersect in a rather small radial region. Qualitatively this is compatible with the factorization result $(10)$ for $P(r, t)$. The MCT results for a HSS are also a superposition of two peaks, one decreasing and the other increasing for $\hat{t}>\hat{t}_{3}$. However in order to reproduce a figure as shown in [14] the theoretical $P^{c}(r)$ as well as $k(r)$ should look similar to the dotted curves in Fig. 1. Experimentally $P^{c}(r)$ is found to exhibit a peak at $r / d \approx 0.19$ and a rather extended wing for $r / d>2 . k(r)$ has a positive peak at $r / d=0.19$ and a broad negative peak around $r / d=1$.

Obviously the MCT introduces a big quantitative error in the spatial correlations for short distances. The position of the first peak of $P(r)$ in [14] is roughly in agreement with the MCT result, but the position of the second peak in $k(r)$ is wrong by a factor 5 . Moreover the experimental peaks show wings extending to greater distances. A reservation applies to this conclusion: it is possible but not studied in detail, that a quantitative MCT analysis of the quoted binary mixture would result in spatial variations closer to the experimentally found ones. It is interesting to note that corresponding molecular dynamics simulations of $\mathrm{CaKNO}_{3}$ show no indication of such double peaks. At present it is not possible to decide whether the identified discrepancy between the MCT result for the HSS and the quoted computer experiment is due to an underestimation of the spatial correlations in the idealized glass transition picture or due to an influence of activated hopping processes on the amplitude $k(r)$. The time dependence of $G(t)$ has to be analyzed in order to decide whether activated hopping processes are to be included or not.

\section{3. $\beta$-relaxation of a soft sphere mixture}

Detaild molecular dynamics studies for a binary soft sphere mixture provided a variety of important results on glassy dynamics. In particular some critical value $\Gamma_{c}$ for the dynamics and some signatures of $\beta$-relaxation in the MCT sense have been identified [14]. Recently [15] arguments have been given that for the mentioned system activated hopping processes should be particularly important. The latter are not incorporated in the simplest version of the MCT. In this section we want to examine whether or not these arguments can be supported by quantitative analysis as far as the dynamics of the $\beta$ relaxation is concerned. It is not possible to study the indicated issue for the $\alpha$-regime at present, since the corresponding master functions have not yet been calculated. The $\beta$ regime can be studied, because the exponent parameter is known: $\lambda=0.73$ [16]. It implies the exponents $a=0.31$ and $b=0.59$. The master functions $g_{+}$are shown in Fig. 3. The $\beta$ region is expected to be particularly extended at the structure factor peak $q_{0}$. So we restrict ourselves to that case. The analysis follows the procedure exercised previously for the HSS [11].

Figure 4 reproduces the data for the tagged particle correlator of the first species and $q=q_{0}$. The full lines are the functions $\Phi_{0}(t)=f_{0}+\left[h_{0} c_{\sigma}\right] g_{-}\left(t / t_{\sigma}\right)$ with $f_{0}=0.68$ and properly chosen scales $\left(h_{0} c_{\sigma}\right), t_{\sigma}$. Except for very short times, where the microscopic dynamics influences the behaviour, there are no deviations between 


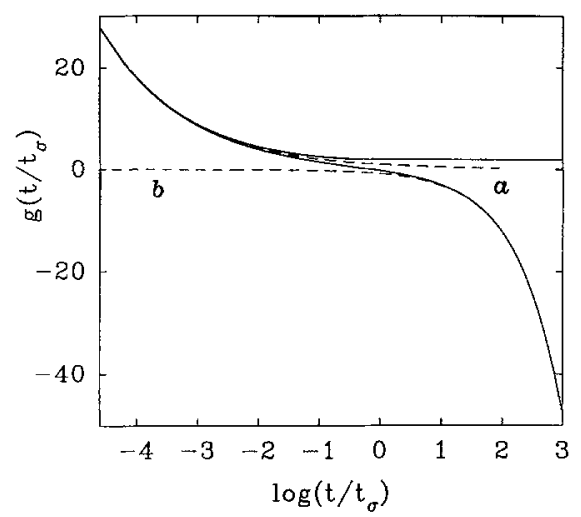

Fig. 3. The upper and lower solid curves are the $\beta$-master functions of the glassy $\left(g_{+}\right)$and the liquid state $\left(g_{-}\right)$respectively for the exponent parameter $\lambda=0.73$. The abscissa shows the rescaled time $\hat{t}=t / t_{\sigma}$. The dashed lines $a$ and $b$ represent the critical decay $\hat{t}^{-a}$ with $a=0.31$ on one hand and the von Schweidler law $-B \hat{t}^{b}$ with $b=0.59$ and $B=0.81$ on the other hand

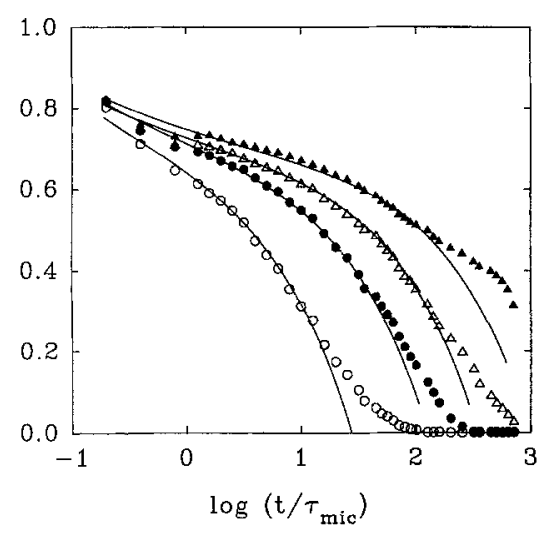

Fig. 4. Experimental relaxation data for the first tagged particle correlator at the peak of the structure factor published in [14]. The four symbols refer to the coupling constants $\Gamma=1.38$ o, $\Gamma=1.43$ $\bullet, \Gamma=1.44 \Delta$ and $\Gamma=1.45 \wedge$. The solid lines demonstrate the theoretical prediction made by $\Phi(t)=f_{0}+\left[h_{0} c_{\sigma}\right] g_{-}(\hat{t})$ with the following values for the fit parameters: $f_{0}=0.68$ for all curves; $\Gamma=1.38$ : $\left(h_{0} c_{\sigma}\right)=0.11, t_{\sigma} / \tau_{\text {mic }}=0.87 ; \Gamma=1.43:\left(h_{0} c_{\sigma}\right)=0.08, t_{\sigma} / \tau_{\text {mic }}=2.35$; $\Gamma=1.44: \quad\left(h_{0} c_{\sigma}\right)=0.06, t_{\sigma} / \tau_{\mathrm{mic}}=3.95 ; \quad \Gamma=1.45:\left(h_{0} c_{\sigma}\right)=0.05$, $t_{\sigma} / \tau_{\text {mic }}=8.34$. In this context $\tau_{\text {mic }}$ refers to the microscopic time scale of the MD-siumulations defined in [14]

fits and data on the short time end of the explored window. At large times the data deviate from the $\beta$-relaxation curves as expected because of the $\alpha$-process. This holds with the possible exception for the coupling constant $\Gamma=1.45$, where the data for the longest times possibly do not fit into the ideal glass transition $\alpha$-relaxation master curves. However, at this coupling the system behaves almost nonergodically, and so one wonders, whether the results really refer to a properly averaged equilibrium correlator. The fit value $f_{0}$ differ by $4 \%$ from the calculated one [15].

Figure 5 presents the fit parameters $\left[h_{0} c_{\sigma}\right]^{2}$ and $t_{\sigma}^{-2 a}$ as functions of $\Gamma$. According to (4) the data should follow straight lines and intersect the abscissa at the same value for $\Gamma_{c}$. This is the case allowing for reasonable uncertainties. Fits of the same quality are obtained [17] for the

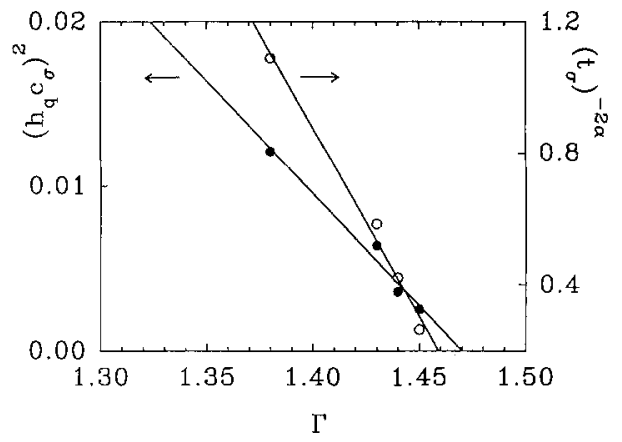

Fig. 5. Fit values $\left(h_{0} c_{\sigma}\right)^{2}$ (full circles) and $\left(t_{\sigma} / \tau_{\text {mic }}\right)^{-2 a}$ (open circles) mentioned at Fig. 4 plotted against coupling constant $\Gamma$. The full lines are linear least square fits through the data

other two correlators published in [14]. Let us emphasize that the scale $t_{\sigma}$ was required to have the same value for the three correlators. From the shown analysis one estimates for the critical coupling: $1.45 \leqq \Gamma_{c} \leqq 1.48$. We have also used the $\beta$-correlators as they follow from the extended theory with hopping effects included. These processes enter the $\beta$-equations as one additional critical parameter $\delta$ [7]. The fits could not be improved by choosing $\delta \neq 0$.

Figure 4, 5 show that the time dependence of the simulation data [14] in the $\beta$-regime is compatible with the MCT for an ideal liquid to glass transition. For $\Gamma=1.45$ the fits of $G(t)$ also work in a time range where $P(r, t)$ shows the onset of the two peak structure as discussed in Chap. 3. This time window is consistent with the explicit observation of the factorization property in the coherent dynamics [14]. For longer times, however, the dynamics cannot be described by the $\beta$-process of the MCT even including hopping effects. The $\alpha$-relaxation must be responsible for the further changing of the two peak structure in $P(r, t)$. It is not yet possible to decide whether the simple version of the MCT can describe the observed incoherent motion in the $\alpha$-regime of a binary mixture.

\section{The $\alpha-\beta$-relaxation pattern of the hard sphere system}

If one analyses $\alpha$-processes, one faces the following problem. For times $t$ very short compared to the $\alpha$-relaxation time $\tau$ the $\alpha$-relaxation scaling laws become invalid because of $\beta$-processes. One will find discrepancies between data and $\alpha$-relaxation MCT results for sufficiently small times. Without a quantitative analysis of the $\beta$-processes one cannot decide easily, whether these discrepancies reflect a short-coming of the theory or whether they merely are caused by the faster glassy relaxation process. Quite a similar problem appears, if one analyses $\beta$-processes. If $t$ exceeds $t_{\sigma}$ by far becoming of the order of $\tau$, the $\beta$ laws $(1,2)$ will fail because of the $\alpha$-process. These are the discrepancies between data and $\beta$-relaxation MCT results as shown for large $t$ in Fig. 4. Without a quantitative analysis of the $\alpha$-process one again is in trouble to decide whether the deviations refer to a defect in the theoretical understanding or whether they are caused by 
the other important glassy relaxation process. A much deeper understanding can be achieved, if one analyses $\alpha$ and $\beta$-processes simultaneously. This is even more evident, if one remembers that the MCT establishes a close link between $\alpha$ - and $\beta$-process. The von Schweidler law is shared by both processes for $t_{\sigma} \ll t \ll t_{\sigma}^{\prime}$ if close to the transition $\sigma \rightarrow-0$ both relaxation processes can be separated well. In this regime, the density autocorrelation function can be expanded asymptotically. The $\alpha$-master function leads to [3]:

$\Phi_{q}(t)=f_{q}^{c}-h_{q}\left(\frac{t}{\tau}\right)^{b}+\delta \Phi_{q}^{\alpha}(t)$ for $\frac{t}{\tau} \rightarrow 0$

$\delta \Phi_{q}^{\alpha}(t)=h_{q}^{(2)}\left(\frac{t}{\tau}\right)^{2 b}-h_{q}^{(3)}\left(\frac{t}{\tau}\right)^{3 b}+O\left[\left(\frac{t}{\tau}\right)^{4 b}\right]$.

The $\beta$-relaxation function $g_{-}$asymptotically for $\frac{t_{\sigma}}{t} \rightarrow 0$ leads to [3]:

$\Phi_{q}(t)=f_{q}^{c}+c_{\sigma} h_{q}\left\{-B\left(\frac{t}{t_{\sigma}}\right)^{b}\right\}+\delta \Phi_{q}^{\beta}(t)$

$\delta \Phi_{q}^{\beta}(t)=c_{\sigma} h_{q}\left\{\left(\frac{B_{1}}{B}\right)\left(\frac{t}{t_{\sigma}}\right)^{-b}+O\left[\left(\frac{t}{t_{\sigma}}\right)^{-3 b}\right]\right\}$

A matching of (11) and (13) yields the known connections between the scales:

$\tau=B^{-\frac{1}{b}} t_{\sigma}^{\prime}=B^{-\frac{1}{b}}|\sigma|^{-\frac{1}{2 b}} t_{\sigma}$.

The von Schweidler law however can be isolated in experimental data only if the corrections $\delta \Phi_{q}^{\alpha}(t)$ and $\delta \Phi_{q}^{\beta}(t)$ are small. If $\alpha$ and $\beta$ time scales are not separated well enough, the corrections $\delta \Phi_{q}^{\beta}(t)$ lead to deviations of the data from the $\alpha$-master curve (11) already for not too small $t / \tau$ where $\delta \Phi_{q}^{\alpha}(t)$ is not yet negligible.

In this section a combined $\alpha-\beta$-relaxation analysis shall be worked out for the mentioned light scattering data of a colloidal suspension [9]. The data have been analyzed before with the MCT results for the HSS separately for the $\alpha$ - [13] and the $\beta$-process [11]. A fit to the whole $\alpha$ $\beta$-process reduces the number of fit parameters by two compared to the $\beta$-analysis of [11] making the comparison between theory and experiment more challenging. Relation (15) connects time scales $t_{\sigma}$ and $t_{\sigma}^{\prime}$ and the $\alpha$ master curve fixes the critical amplitude $h_{q} / f_{q}^{c}$. Compared to [11] we loose the possibility to treat $t_{\sigma}$ and $h_{q}$ as fit parameter.

If $\alpha$ and $\beta$ master functions are known the analysis of experimental data should start with fixing $f_{q}^{c}$ and $\tau_{q}$ in the $\alpha$-regime. $\tau_{q}$ is defined by $\Phi_{q}\left(t / \tau_{q}\right)=f_{q}^{c} / e$ and is connected to $\tau$ via $\tau_{q}=\hat{\tau}_{q}$ where $\hat{\tau}_{q}=1.79$ for $q=q_{0}$ at the peak of the structure factor $S_{q}$ [13]. The deviations of the data from the $\alpha$ master curve are described by $\delta \Phi_{q}^{\beta}(t)(14)$. For the fit only the separation parameter $\sigma$ fixing the scales $t_{\sigma}$ by (15) and $c_{\sigma}$ by (4) can be varied.

Figure $6 \mathrm{a}$ shows the results of the combined fits for packing fractions from $\varphi=0.480$ to $\varphi=0.542$. Starting
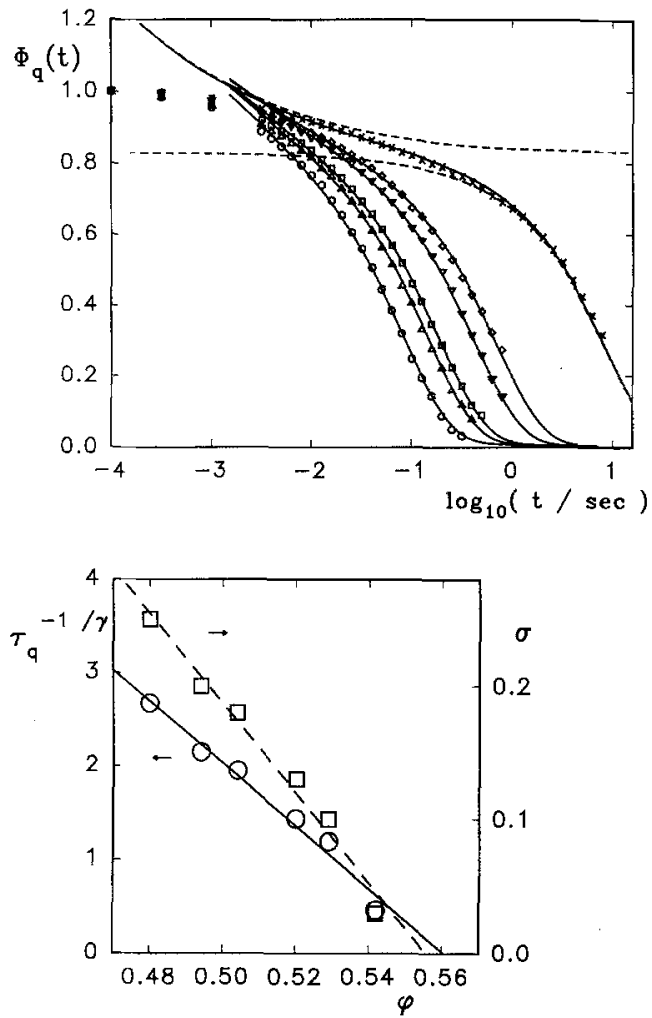

Fig. 6. a Relaxation data of a colloidal suspension for wave vector at the structure factor peak in $S_{q}$ are reproduced from [9], [11]. The symbols $\circ \Delta \square \nabla \diamond \times$ correspond to the packing fractions $\varphi=0.480,0.494,0.504,0.520,0.529$ and 0.542 respectively. The shown theoretical $\alpha-\beta$-relaxation curves are fitted as described in the text. For $\varphi=0.542$ the $\alpha$-master function and the $\beta$-relaxation correction $\delta \Phi_{q}^{\beta}(t)$ decaying onto $f_{q}^{c}=0.83$ are included as dashed lines. The theoretically calculated values $\lambda=0.766, h_{q}=0.30$, $B=1.01$ and $\hat{\tau}_{q}=1.79$ are used, $\mathbf{b}$ Parameters of the $\alpha$ - $\beta$-relaxation fits of Fig. 6a. In a plot of $\left(\tau_{q}\right)^{-1 / y}$ (circles refering to the left scale) and $\sigma$ (squares refering to the right scale) straight lines correspond to the theoretical predictions (8) and (3)

from roughly $t=10^{-2}$ the correlators can be fitted for up to 2.9 decades in time. The $\alpha$-relaxation scale varies by 2 and the $\beta$-relaxation scale by 1.1 decades in the specified $\varphi$-window. In Fig. 6 a the $\alpha$ master function and the correction $\delta \Phi_{q}^{\beta}(t)$ are included as dashed lines for the packing fraction $\varphi=0.542$ closest to the critical packing fraction $\varphi^{c}=0.560$ [9].

Figure $6 \mathrm{c}$ checks the variation of the two fit parameters $\sigma$ and $\tau_{q}$ with packing fraction $\varphi$. The predicted laws (3) and (8) are shown as curves.

The problem of analyzing $\alpha$-relaxation data phenomenologically without properly excluding the $\beta$-relaxation process shall be exemplified for the quoted colloidal data. The full curves in Fig. 7 show fits by the popular Kohlrausch law $\Phi^{K}(t)=f_{K} \exp -(t / \tau)^{\beta}$. For each packing fraction $\varphi$ the three parameters $f_{K}, \tau$ and $\beta$ are chosen to optimize the fit for times beyond the microscopics. The result of the fit is not much worse than the one achieved in Fig. 6a. If one took the ad hoc procedure leading to Fig. 7 seriously, one would arrive at conclusions which are quite different from those suggested by the MCT. The Kohlrausch fit requires a strong increase of the stretching 


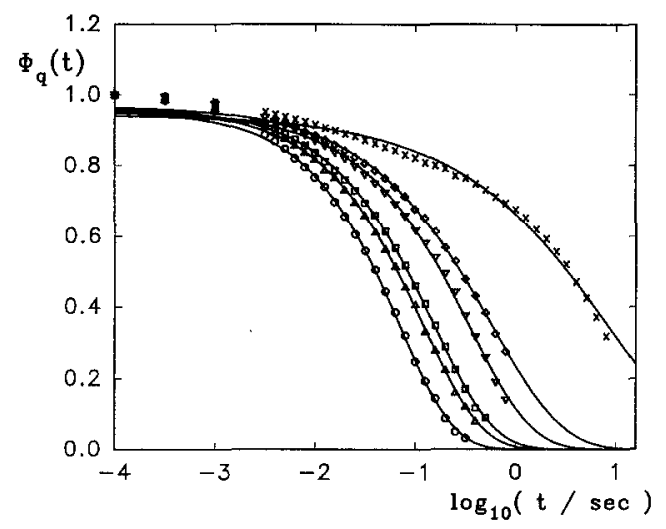

Fig. 7. Relaxation data of the colloidal suspension as shown in Fig. $6 \mathrm{a}$. The solid lines correspond to least square Kohlrausch fits in the same time interval where the $\alpha-\beta$-master curves describe the data. The fit parameters are: for $\varphi=0.480: f^{K}=0.95, \tau=0.070$ and $\beta=0.80$; for $\varphi=0.494: f^{K}=0.96, \tau=0.119$ and $\beta=0.74$; for $\varphi=0.505: f^{K}=0.96, \tau=0.151$ and $\beta=0.72 ;$ for $\varphi=0.520$ : $f^{K}=0.95, \tau=0.340$ and $\beta=0.69$; for $\varphi=0.529: f^{K}=0.97, \tau=0.559$ and $\beta=0.60$; for $\varphi=0.542: f^{K}=0.94, \tau=8.33$ and $\beta=0.49$

exponent $\beta$ from 0.49 to 0.80 if the packing fraction decreases from 0.542 to 0.480 . This implies a violation of the superposition principle 7 .

\section{The asymptotic region for the hard sphere system}

Formulae $(1-6,11-14)$ are examples for asymptotic solutions of mode coupling equations. They deal with the leading singular contribution in the limit $\sigma \rightarrow 0$. If one compares such results quantitatively with experiments, one faces the following problem. One usually does not know the range of validity of the theoretical results and thus one may interpret data with asymptotic formulae for such large $\sigma$, where the latter are invalid. In this section we want to discuss a special example as illustration of the specified problem, namely the Debye-Waller factor $f_{q}$ for the HSS. This is a relevant case, since it can be measured for hard sphere colloids [9].

From $(1-3,6)$ one gets the asymptotic expression

$f_{q}^{a s}=f_{q}^{c}+\tilde{h}_{q} \sqrt{\varepsilon}, \quad \varepsilon=\frac{\varphi-\varphi_{c}}{\varphi_{c}}$.

The general solution for $f_{q}$ follows from the implicit equation

$\frac{f_{q}}{1-f_{q}}=\mathscr{F}_{q}\left(f_{q}\right)$

where the mode coupling functional $\mathscr{F}_{q}$ is given in terms of the structure factor $S_{q}$ [10]. The equation (17) is solved by iteration, whereby $\varphi_{c}$ and $\tilde{h_{q}}=\tilde{C} h_{q}, \tilde{C}=2.26$ are obtained as by products [12].

Figures $8 \mathrm{a}$ and $9 \mathrm{a}$ exhibit $f_{q}$ as full curves and $f_{q}^{a s}$ as dashed ones for a representative set of $\varepsilon$ values. Figure $8 \mathrm{a}$ is based on the Verlet-Weiss (VW) approximation for $S_{q}$ and Fig. 9a uses the Percus-Yevick (PY) approximation.

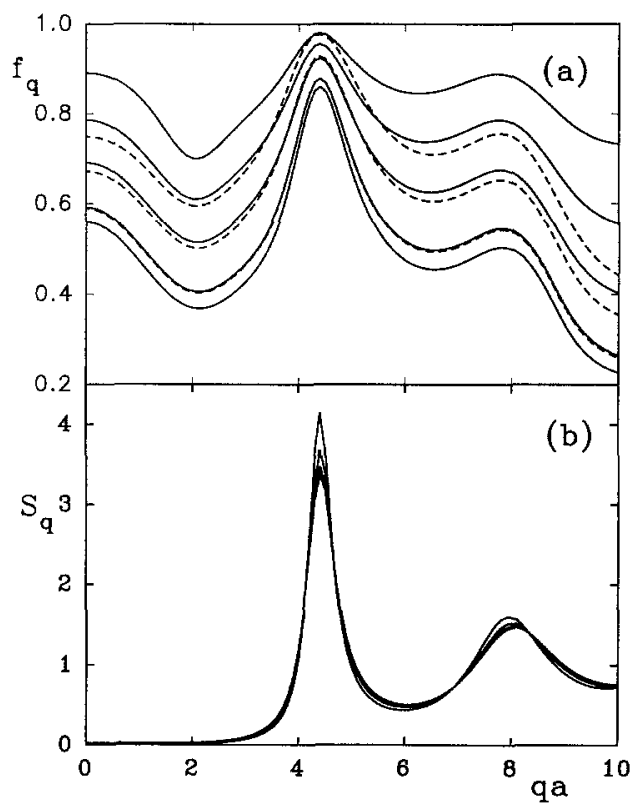

Fig. 8. a Debye Waller factor $f_{q}$ of the HSS for four different separations $\varepsilon=\left(\varphi-\varphi_{c}\right) / \varphi_{c}, \varepsilon=0,7.0 \times 10^{-4}, 0.010,0.029$ and 0.067 as function of $q$ using the $\mathrm{VW}$-structure factor as input (solid lines). $a=\sqrt[3]{(3 / 4 \pi n)}$ is the averaged interparticle distance. The dashed lines show the asymptotic law $f_{q}=f_{q}^{c}+2.26 h_{q} \sqrt{\varepsilon}$ for the same separations $\varepsilon$. The critical packing fraction is $\varphi_{c}=0.525$. b VWstructure factor of the HSS for the same packing fractions

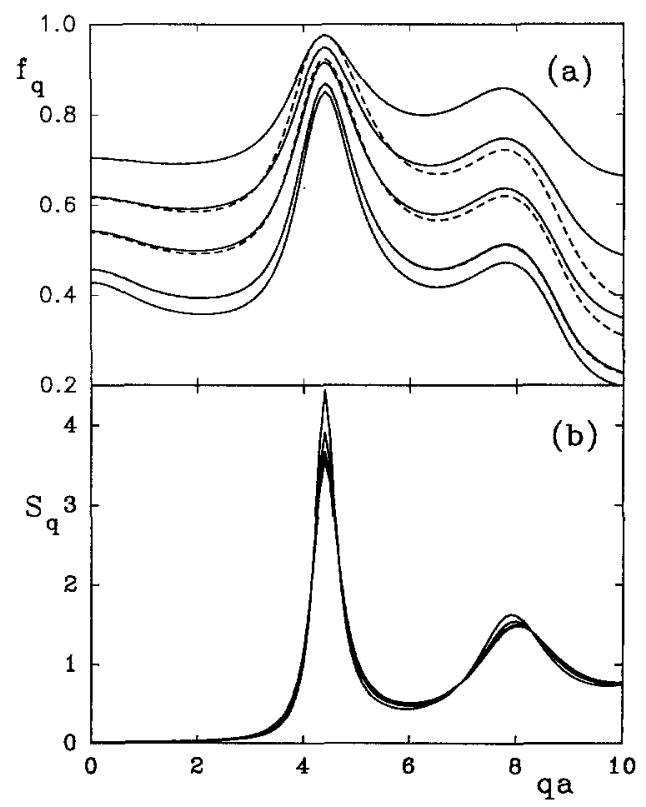

Fig. 9. a Debye Waller factor $f_{q}$ of the HSS for four different separations $\varepsilon=\left(\varphi-\varphi_{c}\right) / \varphi_{c}, \varepsilon=0,7.0 \times 10^{-4}, 0.010,0.029$ and 0.066 as function of $q$ using the PY-structure factor as input (solid lines). $a=\sqrt[3]{(3 / 4 \pi n)}$ is the averaged interparticle distance. The dashed lines show the asymptotic law $f_{q}=f_{q}^{c}+2.28 h_{q} \sqrt{\varepsilon}$ for the same separation $\varepsilon$. The critical packing fraction is $\varphi_{c}=0.516 . \mathrm{b} \mathrm{PY}$-structure factor of the HSS for the same packing fractions 


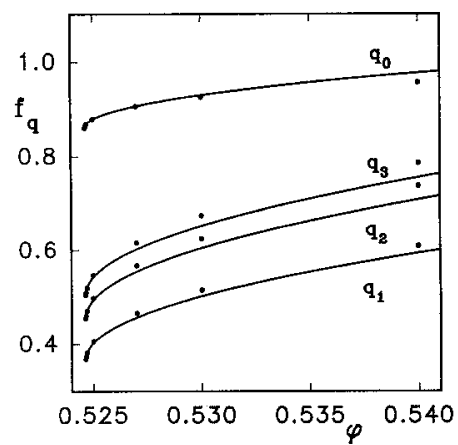

Fig. 10. Debye Waller factor of the HSS using the VW structure factor as function of packing fraction $\varphi$ for $q_{1}=2.1 / a, q_{0}=4.4 / a$, $q_{2}=6.5 / a, q_{3}=7.8 / a$ (solid circles). The solid lines are the asymptotic laws $f_{q_{i}}=f_{q_{i}}^{c}+2.26 h_{q_{i}} \sqrt{\varepsilon}$

The structure factors are shown in Figs. $8 \mathrm{~b}, 9 \mathrm{~b}$. The critical packing fraction $\varphi_{c}$ is found to differ by less than two percent for the two structure factors; $\varphi_{c}=0.516$ if the PY and $\varphi_{c}=0.525$ if the VW approximation is used.

One notices that there are differences, especially for small $q$-vectors, between the results using the VW- and the PY-structure factor. These differences are due to the different behaviour of the structure factor in the hydrodynamic regime and at the first peak at $q=q_{0}$. From Figs. 8a and 9a one concludes that the MCT result for $f_{q}$ except for wave vectors close to the primary peak position $q \approx q_{0}$ is quite sensitive to the correct choice of the structure factor. The correct $S_{q}$ for a given system has to be found before the MCT equations for $f_{q}$ can be checked in the sensitive $q$-range.

The deviations from the asymptotic $\beta$-relaxation regime depend on the choice of the structure factor and are also somewhat $q$-dependent. In general a relatively large range of validity is found at the peak of the structure factor. There, if one assumes an experimental accuracy $\Delta f \approx 0.02$ in the measurement of $f_{q}$, one finds detectable deviations from asymptotic behaviour for $\varepsilon>0.03$. This is also seen in Fig. 10 where $f_{q}=f_{q}(\varphi)$ is shown for four wave vectors using the VW structure factor. For the largest $f_{q}$ at $q=q_{0}$ the asymptotic curve starts to exceed the correct one at $\varphi \geqq 0.53$. At $\varphi=0.54$ the uncertainty of $f_{q}$ is larger than 0.02 . In general for other $q$-vectors the range of validity is of the same size or somewhat smaller. For $q$ larger than the position of the second minimum in $f_{q}$ the asymptotic regime is considerably smaller, $\varepsilon<0.01$ has to hold. Below the peak of $S_{q}$, i.e. for $q<q_{0}$, VW and PY structure factor lead to different deviations from the asymptotic law.

We thank W. Van Megen, S. M. Underwood and P. N. Pusey for the permission to study their $f_{q}$-measurements prior to publication; their findings motivated the discussion in Sect. 5. We acknowledge gratefully helpful comments by J.L. Barrat.

\section{References}

1. Wong, J., Angell, C.A. : Glass: Structure by spectroscopy. Basel: Marcel Dekker 1976

2. Götze, W.: Liquids, freezing and the glass transition. Hansen, J.P., Levesque, D., Zinn-Justin, J. (eds.), p. 287. Amsterdam: North Holland 1991

3. Götze, W.: Amorphous and liquid materials. Lüscher, E. (ed.), p. 34. Dordrecht: Martinus Nijhoff 1987

4. Götze, W., Sjögren, L.: J. Phys.: Condensed Matter 1, 4183 (1989)

5. Götze, W.: J. Phys.: Condensed Matter 2, 8485 (1990)

6. Sjögren, L.: Basic features of the glassy state. Colmenero, J., Alegria, A. (eds.), p. 137. Singapore: World Scientific 1990; Sjögren, L.: J. Phys.: Condensed Matter 3, 5023 (1991)

7. Götze, W., Sjögren, L.: Z. Phys. B - Condensed Matter 65, 415 (1987)

8. Pusey, P.N.: Liquids, freezing and the glass transition. Hansen, J.P., Levesque, D., Zinn-Justin, J. (eds.), p. 763. Amsterdam: North Holland 1991

9. Van Megen, W., Pusey, P.N.: Phys. Rev. A43, 5429 (1991)

10. Bengtzelius, U., Götze, W., Sjölander, A.: J. Phys. C: Solid State Phys. 17, 5915 (1984)

11. Götze, W., Sjögren, L.: Phys. Rev. A43, 5442 (1991)

12. Barrat, J.L., Götze, W., Latz, A.: J. Phys.: Condensed Matter 1, 7163 (1989)

13. Fuchs, M., Hofacker, I., Latz, A.: (preprint, August 1991)

14. Roux, J.N., Barrat, J.L., Hansen, J.P.: J. Phys.: Condensed Matter 1, 7171 (1989); Barrat, J.L., Roux, J.N., Hansen, J.P.: Chem. Phys. 149, 197 (1990)

15. Barrat, J.L., Roux, J.N.: J. Non-Cryst. Solids 131-133, 255 (1991)

16. Barrat, J.L., Latz, A.: J. Phys.: Condensed Matter 2, 4289 (1990)

17. Hildebrand, S.: Diplomarbeit, TU München. (unpublished, 1991) 